Original Article

\title{
PHYSICOCHEMICAL CHARACTERIZATION AND EVALUATION OF TELMISARTAN: HYDROXYPROPYL- $\beta$-CYCLODEXTRIN: TWEEN 80 INCLUSION COMPLEX
}

\author{
POONAM ARORA ${ }^{1 *}$, JASPREET SINGH ${ }^{1}$, RENU CHADHA ${ }^{2}$ \\ ${ }^{1}$ GHG Khalsa College of Pharmacy, Gurusar Sadhar, Ludhiana, Punjab, ${ }^{2}$ University Institute of Pharmaceutical Sciences, Panjab University, \\ Chandigarh, Punjab India \\ Email: poonam803@yahoo.com
}

Received: 10 Apr 2017 Revised and Accepted: 13 Jul 2017

\begin{abstract}
Objective: The present work was aimed to study the effect of the ternary component on complexation efficiency of cyclodextrins towards telmisartan which is a poorly soluble anti-hypertensive agent.

Methods: The elucidation of inclusion complexation of telmisartan (TEL) with hydroxypropyl- $\beta$-cyclodextrin (HP- $\beta$-CD) in the presence and absence of tween 80 was done by investigating their interactions in solid and solution state. The solid state characterization was performed using differential scanning calorimetry (DSC), powder X-ray diffraction studies (PXRD) fourier transform infra-red spectroscopy (FTIR) studies. The host guest stoichiometry was confirmed in solution state by proton nuclear magnetic resonance $\left({ }^{1} \mathrm{HNMR}\right)$ and solution calorimetry studies. The improvement in solubility was evaluated through dissolution studies, which was further confirmed by in vivo studies.
\end{abstract}

Results: In solution state, the phase solubility studies indicated 1:1 stoichiometry between TEL and HP- $\beta$-CD both in presence and absence of tween 80 . The NMR and molecular modelling studies indicated the insertion of N-methyl benzimidazole and biphenyl carboxylate regions of TEL into HP- $\beta$-CD cavity suggesting the coexistence of two $1: 1$ complexes in equilibrium with each other. The stability constants, $\mathrm{K}_{1}$ (imidazole region of TEL-CD) and $\mathrm{K}_{2}$ (biphenyl acetic acid region of TEL-CD), were enhanced in the presence of tween 80 as evident by the higher value of stability constants. Efficacy of ternary complex was established by a significant decrease in the systolic blood pressure of deoxycorticosterone acetate (DOCA) induced hypertensive rats.

Conclusion: It can be concluded that solubility of TEL was increased by encapsulation with HP- $\beta$-CD. Tween 80 further increased the complexation efficiency and decreased the bulk of cyclodextrin.

Keywords: Cyclodextrin, Telmisartan, Ternary complex, Stability, Tween 80, Calorimetry, DOCA

(C) 2017 The Authors. Published by Innovare Academic Sciences Pvt Ltd. This is an open access article under the CC BY license (http://creativecommons.org/licenses/by/4.0/) DOI: http://dx.doi.org/10.22159/ijpps.2017v9i9.19058

\section{INTRODUCTION}

The supramolecular chemistry deals with supramolecular architectures which are based on molecular recognition between complementary host-guest and self-assembly into purely hydrogen bonded networks without establishing covalent bonds between interacting species [1]. Over the past few decades, among the majority of hosts, the cyclodextrins due to various desirable attributes like absorbability, biodegradability and encapsulation ability of a wide variety of guest molecules with suitable polarity and dimensions, seems to be the most important host to form inclusion complexes [2-4].

Cyclodextrins are cyclic oligosaccharides in which several D-glucose units are linked by $\alpha-1,4$ glycosidic bonds providing hydrophobic cavities to accommodate appropriately sized guest molecules $[5,6]$. The molecular encapsulation of lipophilic drugs with appropriate cyclodextrin lead to improved physicochemical properties of drug and consequently facilitating safe and efficient delivery of drugs which makes cyclodextrins to be widely used in pharmaceutical industry with the aim of enhancing drug aqueous solubility [7-13]. However, an increase in formulation volume represents a limiting factor in the applicability of cyclodextrin due to the inherent toxicity as well as the cost associated with cyclodextrin [14]

The use of small amounts of water soluble polymer increases the complexation efficiency leading to decreased formulation bulk and cost. Although the mechanism involved in increasing the complexation efficiency is not fully understood, however, it is believed that the addition of a ternary component to the binary complex leads to improved macromolecular self-assembly [15-22].

TEL is a poorly soluble angiotensin II receptor antagonist which is used as an anti-hypertensive agent. This drug belongs to BCS class II category and thus is poorly water soluble. It is necessary to improve the solubility of such agents in order to increase the bioavailability.

The truncated shape of cyclodextrins makes them attractive alternatives for complexation of the drug molecule. Although the complementary effect of cyclodextrins complexation on TEL solubility and dissolution rate has also been demonstrated previously [23-26], however, the effect of the third component on inclusion complexation, binding parameters, as well as pharmacodynamic evaluation, has not been reported.

Therefore the present study investigates the interaction of telmisartan (TEL) with hydroxyl-propyl- $\beta$-cyclodextrin (HP- $\beta$-CD) in the absence and presence of tween 80 as third component in both solution (phase solubility, proton nuclear magnetic resonance (1HNMR), solution calorimetry) and solid (differential scanning calorimetry (DSC), powder X-ray diffraction studies (PXRD), fourier transform infrared spectroscopy (FTIR)) phases and is also focused on determining the thermodynamic parameters of binary and ternary complexes, exact stoichiometry as well as the geometry of the complexation using molecular modeling.

\section{MATERIALS AND METHODS}

\section{Materials}

TEL was obtained as a gift sample from Ind-swift Laboratories Ltd. (Panchkula, India). $\beta$-cyclodextrin ( $\beta$-CD) and hydroxyl-propyl- $\beta$ cyclodextrin (HP- $\beta-\mathrm{CD}$ ) were purchased from Hi-media Lab. Pvt Ltd. (Mumbai, India), and methyl-- $\beta$-cyclodextrin (M- $\beta$-CD) was purchased from Sigma-Aldrich, Co. (St. Louis, USA). All other chemicals were of analytical grade.

\section{Preparation of complexes}

Binary complexes of TEL with HP- $\beta$-CD were prepared in molar ratio of $1: 1$ by (a) physical mixing (PM) mixing both (TEL and HP- $\beta$-CD) 
with a spatula for $25 \mathrm{~min}$ and then sieved through 120 \# and packed in airtight container; (b) kneading $(K N)$ the $\mathrm{CD}$ in a mortar with small quantity of water to obtain a homogeneous paste, TEL was then added slowly while grinding and to facilitate the dissolution of TEL into CD paste, a small quantity of methanol was added. The mixture was then grounded for $6 \mathrm{~h}$. During this process, an appropriate quantity of water was added to the mixture to maintain the desired consistency. The pastes were dried in oven at $45-50^{\circ} \mathrm{C}$ for $24 \mathrm{~h}$. The dried complexes were pulverized and then sieved through 120 \#.

Ternary complexes were prepared by adding tween $80(0.1 \% \mathrm{v} / \mathrm{v})$ to a solution of drug and selected cyclodextrin in water. This solution was autoclaved at $120^{\circ} \mathrm{C}$ for half an hour to activate water soluble polymer through heating for enhanced drug solubilization $[27,28]$ and then grounded for $6 \mathrm{~h}$. An appropriate quantity of water was added to the mixture to maintain the desired consistency. The pastes were dried in an oven at $45-50{ }^{\circ} \mathrm{C}$ for $24 \mathrm{~h}$. The dried complexes were pulverized and then sieved through 120 \#.

\section{Equilibrium phase solubility analysis of TEL in presence and} absence of tween 80

Equilibrium phase solubility studies were carried out according to the method described by Higuchi and Connors [29]. TEL, in constant amounts (5 mg) exceeding its solubility, was transferred to conical flasks containing $10 \mathrm{ml}$ of aqueous solution of HP- $\beta-C D$ at various molar concentrations $(0-$ 10 mmoles) both in absence and presence of $0.1 \% \mathrm{v} / \mathrm{v}$ Tween 80 . The mixtures were firstly dispersed by vortex shaker for $2 \mathrm{~min}$, and then oscillated by rotary shaker (MSW-275 Macro scientific works, Delhi) at 37 ${ }^{\circ} \mathrm{C}$ for $24 \mathrm{~h}$. The time duration was fixed based on pilot experiment and found to be sufficient to achieve equilibrium of mixture. After reaching equilibrium, the samples were withdrawn, filtered through $0.45 \mu \mathrm{m}$ membrane filter and analyzed spectrophotometrically (Perkin Elmer precisely, Lambda 25, UV/VIS spectrophotometer) at $\lambda \max (297 \mathrm{~nm})$.

\section{Characterization in solid state}

\section{Differential scanning calorimetry (DSC)}

DSC measurements were carried out on DSC (Q20, TA Instruments Waters LLC, USA) which was calibrated using pure indium. The samples were weighed $(3 \mathrm{mg})$ in aluminium pans and scanned at a heating rate of $10^{\circ} \mathrm{C}$ in the temperature range from 50 to $350^{\circ} \mathrm{C}$. Dry nitrogen with a flow rate of $50 \mathrm{ml} / \mathrm{min}$ was used as purge gas.

\section{Fourier transform infrared spectroscopy (FT-IR)}

The FT-IR spectra of TEL, its physical mixture, binary and ternary complexes over the range $4000 \mathrm{~cm}^{-1}-450 \mathrm{~cm}^{-1}$ were obtained on FTIR spectrometer (Mode spectrum RXI, Perkin Elmer, England). A manual press was used to make the pellets of the samples.

\section{X-Ray powder diffraction (XRPD)}

Powder diffraction patterns were recorded on an X-ray diffractometer (XPERT-PRO, PAN alytical, Netherlands) with $\mathrm{Cu}$ as tube anode. The diffractograms were recorded under the following conditions: voltage $40 \mathrm{kV}, 35 \mathrm{~mA}$, angular range five and fixed divergence slit.

\section{Characterization in solution state}

\section{Nuclear magnetic resonance (NMR) spectroscopy}

Bruker Advance II 400 NMR spectrometer operating at $400 \mathrm{~Hz}$ was used for studying proton NMR spectra of inclusion complexes and pure drug in deuterated dimethyl sulfoxide.

\section{Solution calorimetry}

Isoperibol solution calorimeter (ISC) model 4300 (Calorimetry Science Corporation, Utah, USA) was used for thermal measurements. It is a semi-adiabatic calorimeter with temperature resolution after noise reduction, close to $1 \mathrm{~K}$, which corresponds to a heat resolution of $1-4 \mathrm{~mJ}$ in a $25 \mathrm{ml}$ buffer $(\mathrm{pH} 7)$ reaction vessel. The details are given in our previous papers [31, 31]. The performance of the system was tested by measuring the enthalpy of solution of potassium chloride $(17.301 \mathrm{~kJ} / \mathrm{mol})$ in triple distilled water, which is in good agreement with a known enthalpy of solution of $17.322 \mathrm{~kJ} / \mathrm{mol}$. The precision of any individual measurement was better than $\pm 0.03 \mathrm{~kJ} / \mathrm{mol}$ for three consecutive experiments.

\section{Dissolution study}

The dissolution profiles of the TEL, its binary and ternary complexes were obtained using USP dissolution rate type II apparatus at $37 \pm 0.5^{\circ} \mathrm{C}$. Dissolution media consisted of $900 \mathrm{ml}$ of phosphate buffer ( $\mathrm{pH}$ 7.4), previously filtered, degassed, and maintained at $37 \pm 0.5{ }^{\circ} \mathrm{C}$. The stirring speed was set at $50 \mathrm{rpm}$. The amount of inclusion complexes added was equivalent to $40 \mathrm{mg}$ of TEL. The aliquots of $5 \mathrm{ml}$ were withdrawn at regular time intervals and analyzed till the absorbance of the solution attains a constant value. At each sampling time, an equal volume of fresh medium was added and the correction for the cumulative dilution was calculated. Each dissolution study was performed on duplicate batches.

\section{In vivo studies-pharmacodynamic activity}

Evaluation of the antihypertensive activity of TEL and its binary and ternary complexes was performed in DOCA salt induced hypertensive rats.

4-5 w old albino rats (200-300 g) were procured and were housed at a relative humidity of $65 \pm 2 \%$, temperature of $23 \pm 0.5^{\circ} \mathrm{C}$ with a 12 $\mathrm{h}$ light/dark cycle. They were provided with standard pellet diet and water ad libtum. Experiments were performed as per guidelines of the committee for the purpose of the Control and Supervision on Experiments on Animals (CPCSEA). The experimental protocol was approved by Institutional Animal Ethics Committee (Approval No IAEC/477 dated 19/11/2013).

Animals were divided into 6 groups and each group comprised of 6 animals $(n=6)$.

Group I and II (Control group): These rats were vehicle treated. They were orally fed with distilled water daily and cotton seed oil $(0.1$ $\mathrm{ml} / 100 \mathrm{~g}$ ) was injected subcutaneously twice a week for $6 \mathrm{w}$. After $6 \mathrm{w}$ HP- $\beta$-CD and HP- $\beta$-CD: tween $80(0.1 \% \mathrm{v} / \mathrm{v})$ (blank for complexes) suspended in $0.5 \%(\mathrm{w} / \mathrm{v})$ sodium carboxymethyl cellulose (CMC) suspension was administered orally by gavage to group I and II rats.

Group III (DOCA blus 1\%(w/v) $\mathrm{NaCl}$ treated rats: This group received DOCA and $1 \%(\mathrm{w} / \mathrm{v}) \mathrm{NaCl}$ solution for $6 \mathrm{w}$. After the induction of hypertension $(6 \mathrm{w})$ this group received a single oral dose therapy of HP $\beta$-CD: tween $80(0.1 \% \mathrm{v} / \mathrm{v})$ suspension in CMC.

Group IV: This group received DOCA and 1\% (w/v) NaCl solution for 6 w. After the induction of hypertension $(6 \mathrm{w})$ this group received a single oral dose therapy of $10 \mathrm{mg} / \mathrm{kg}$ of TEL in $0.5 \%(\mathrm{w} / \mathrm{v}$ ) CMC suspension.

Group V (DOCA plus 1\% (w/v) NaCl followed by TEL: HP $\beta$-CD complex: Similar to group IV, this group received DOCA and $1 \%$ $(\mathrm{w} / \mathrm{v}) \mathrm{NaCl}$ solution for $6 \mathrm{w}$ followed by a single oral dose therapy of TEL: HP- $\beta$-CD complex equivalent to $10 \mathrm{mg} / \mathrm{kg}$ TEL.

Group VI (DOCA plus $1 \%(w / v) ~ N a C l$ followed by TEL: HP- $\beta-C D$ : tween $80(0.1 \% \mathrm{v} / \mathrm{v})$ complex: Similar to group IV and V, group VI received DOCA and 1\%(w/v) NaCl solution for $6 \mathrm{w}$ followed by a single oral dose therapy of TEL-HP- $\beta$-CD-tween $80(0.1 \% \mathrm{v} / \mathrm{v})$ complex equivalent to $10 \mathrm{mg} / \mathrm{kg}$ TEL.

Changes in systolic blood pressure of all the groups were recorded at different time intervals $(0,1,2,6,8,12$ and $24 \mathrm{~h})$, by the indirect tail cuff method using the blood pressure recorder Cat. 8006, Ug0 (Basile, Biological Research Apparatus, Italy) to monitor the efficacy and potency of prepared complexes in comparison to the control groups I and II.

\section{Statistical analysis}

Data were expressed as mean $\pm \mathrm{SD}$. One way analysis of variance (ANOVA) was used for each parameter, followed by Tukey's test. $\mathrm{P}<0.01$ is considered statistically significant.

\section{RESULTS AND DISCUSSION}

\section{Phase solubility studies}

\section{Effect of cyclodextrins on aqueous solubility of TEL}

The elucidation of phase solubility profile is the fundamental technique to explain the host-guest interaction because it gives not only the solubilizing ability of the host molecules but also the stochiometric ratio of the components of the complex in which they are associated [30]. The phase solubility studies of TEL were carried out with $\beta-C D, H P-\beta-C D$ and 
M- $\beta$-CD in triple distilled water at $37{ }^{\circ} \mathrm{C}$ for $24 \mathrm{~h}$ and their respective phase solubility profiles for the complex formation are shown in fig. 1. It was observed from the phase solubility diagram results that the type of CD used influences greatly the TEL solubility.

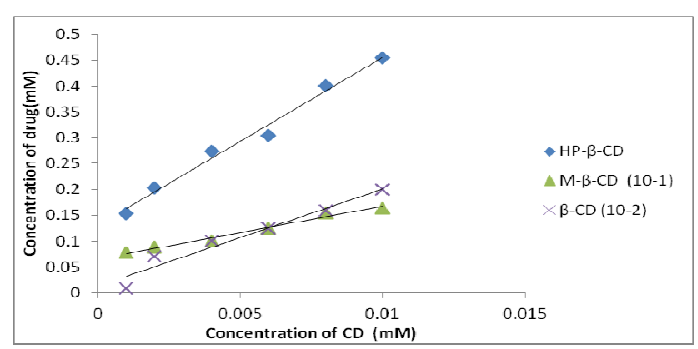

Fig. 1: Phase solubility profiles of telmisartan with $\beta$ cyclodextrin, hydroxyl-propyl- $\beta$-cyclodextrin and methyl- $\beta$ cyclodextrin in triple distilled water

The aqueous solubility of TEL increased linearly as a function of cyclodextrin concentration over range under study, indicating the formation of soluble inclusion complexes with 1:1 stoichiometry. Given the CD used for the binary complexes formation, HP- $\beta$-CD showed superior solubilizing and complexing ability toward TEL and therefore this derivative of $\beta$-CD was selected for further studies.

\section{Screening of the third component}

The use of the third component as a strategy for significant reduction in the amount of cyclodextrin in desired formulations is well established, however, a few reports are also available showing the adverse effect of the added polymer on solubility [33]. Therefore, cautious screening of the third component is very important. Various solubilizing agents were tried to access their efficiency in increasing the solubilizing effect of cyclodextrin. The best performance was shown by tween $80(0.1 \% \mathrm{v} / \mathrm{v})$ out of the various additives (polyvinylpyrrolidone (PVP), hydroxypropyl methyl cellulose (HPMC), polyethylene glycol (PEG) and poloxamer) for increasing the solubilizing efficacy of cyclodextrin (fig. 2). Therefore, it was adjudged to use tween 80 as the hydrophilic additive in the ternary complex of TEL. Although the exact mechanism of interaction of the polymers with binary complexes is not clear, however, it is believed that the hydropillic polymer forms supramolceular aggregate with both drug and binary complex. These polymers aid in the wetability of particles, resulting in an accelerated dissolution and increased the amount of drug delivered in vitro.

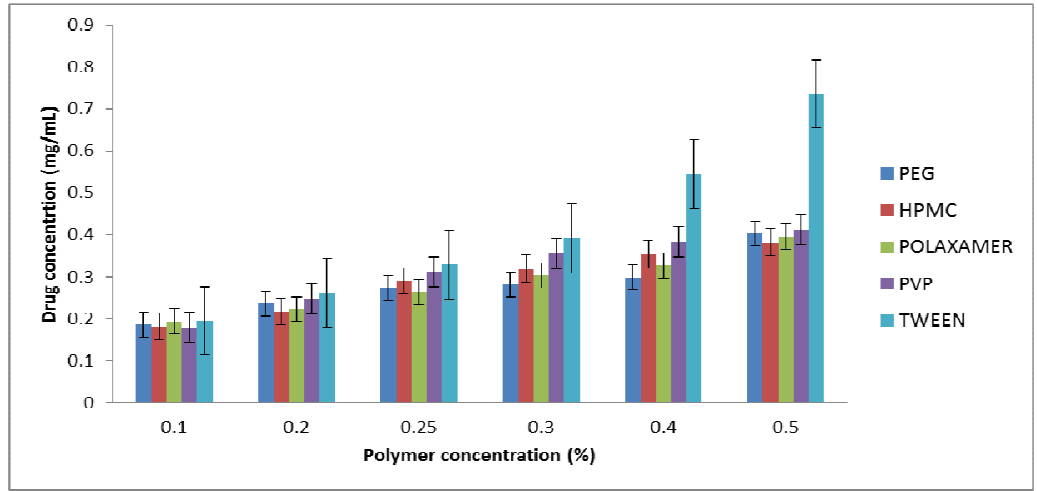

Fig. 2: Effect of different additives on solubility of telmisartan in the presence of hydroxyl-propyl- $\beta$-cyclodextrin, significant increase in solubility of ternary system as compared to binary system $(\mathrm{P} \leq \mathbf{0 . 0 0 5})$

\section{Solubility of TEL in binary and ternary systems}

Many workers have shown that complexation with CDs particularly 2 -HP- $\beta$-CD leads to improved solubility of TEL $[23,26]$. However, the effect of the ternary component has not been studied. In the present study, it was found that the solubility of TEL was 2.86 and 0.46 mmoles L $^{-1}$ at a concentration of 10 mmoles $\mathrm{L}^{-1} 2$-HP- $\beta$-CD with and without $0.1 \% \mathrm{v} / \mathrm{v}$ tween 80 respectively (fig. 3 ). This leads to $\sim 6.2$ fold increase in solubility of TEL on switching over from binary system to ternary system. However, the solubility of TEL in

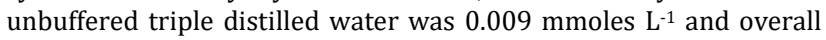
there is several times increase in solubility of TEL in drug: HP- $\beta$-CD $\left(10\right.$ mmoles $\left.\mathrm{L}^{-1}\right)$ :tween $80(0.1 \% \mathrm{v} / \mathrm{v})$ system in comparison to solubility in unbuffered water. Such a synergistic effect can be explained assuming a tween 80 induced higher complexation efficiency of HP- $\beta$-CD towards TEL as compared to without tween 80 . The linear phase solubility curve in the presence of $0.1 \% \mathrm{v} / \mathrm{v}$ of tween 80 was also found to be $A_{L}$ type. The addition of tween 80 does not seem to change the stoichiometry in the complexes (1:1) as the slope still continues to be less than one. This led to the preparation of ternary inclusion complexes containing the drug and cyclodextrin in the presence of $0.1 \%(\mathrm{v} / \mathrm{v})$ tween 80.

\section{Molecular modelling studies}

The molecular modelling studies predicted the existence of two types of complexes with 1:1 stoichiometry and equal stability which can coexist in solution phase (fig. 4). Therefore, the solid binary and ternary complexes were prepared and characterized keeping in mind the $1: 1$ stoichiometry.

\section{Differential scanning calorimetry (DSC)}

The DSC thermograms of TEL, HP- $\beta-C D$ and both binary and ternary complexes along with the physical mixture are given in fig. 5 . The endothermic peak can be detected at $270{ }^{\circ} \mathrm{C}$ in the DSC thermogram of TEL while a broad endotherm corresponding to desolvation was observed in HP- $\beta$-CD thermogram. The thermograms of both binary and ternary complexes lacked the endothermic peaks arising from individual components and showed a broad transition extending from 60 to $120^{\circ} \mathrm{C}$ in a binary system and 80 to $160{ }^{\circ} \mathrm{C}$ for the ternary complex. The disappearance of the endothermic peaks suggests insertion of the guest molecule into the $\mathrm{CD}$ cavity. The thermograms of physical mixture resembled the combination of curves of individual components.

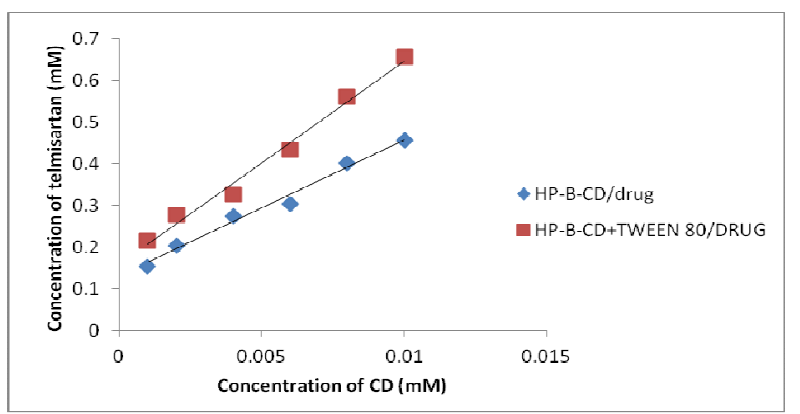

Fig. 3: Phase solubility diagram of telmisartan with hydroxylpropyl- $\beta$-cyclodextrin in the absence and presence of tween 80 


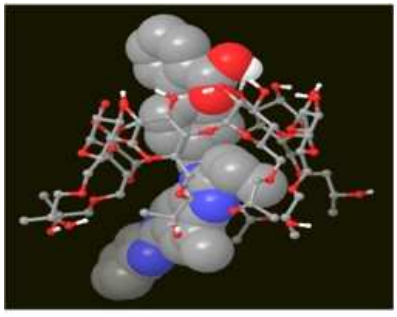

Case-1

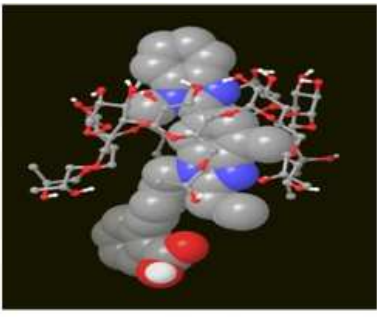

Case-2
Fig. 4: Orientation of complex on inclusion of telmisartan into hydroxyl-propyl- $\beta$-cyclodextrin cavity

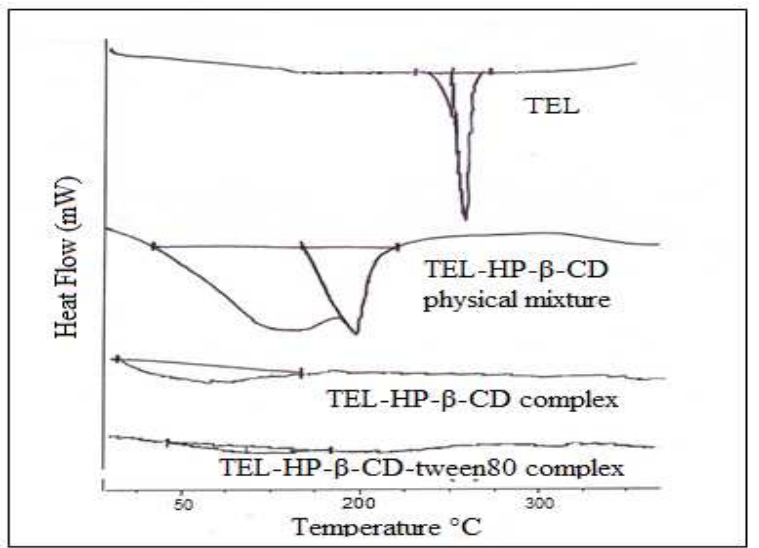

Fig. 5: DSC curves of telmisartan, its physical mixture and binary and ternary complex

\section{X-ray powder diffraction (XRPD)}

The characteristic diffraction peaks relevant to crystalline TEL appear at $2 \theta=6.84,14.28$ and 22.38 (fig. 6). The diffraction pattern of the investigated PM of TEL and HP- $\beta$-CD at 1:1 molar ratios is apparently superposition of diffraction pattern of individual components, indicating that drug maintained its crystallinity in its respective physical mixture. The binary system presented a diffraction pattern with fewer peaks of lower intensity and the results are comparable with the XRPD pattern shown by Lofftson et al. [26]. Whereas it was no longer possible to distinguish the characteristic peaks of the drug in the ternary system (TEL: HP- $\beta$ -
CD: tween 80) which showed a modified and almost halo pattern suggesting the formation of amorphous inclusion complex (fig. 6).

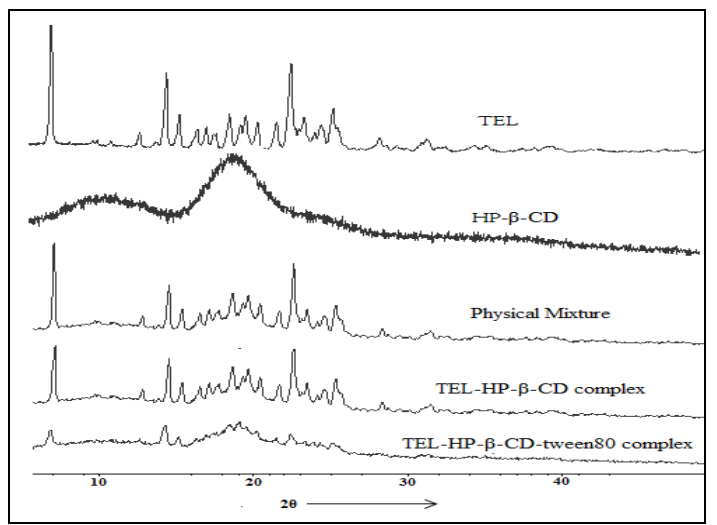

Fig. 6: X-ray diffraction pattern of telmisartan, its physical mixture and binary and ternary complex

\section{Fourier transform infrared spectroscopy (FT-IR)}

In the FT-IR spectra of TEL distinct changes were observed in characteristic IR peaks which occurred at $1696 \mathrm{~cm}^{-1}$ for the carbonyl group $\mathrm{C}=\mathrm{O}$ and $3416 \mathrm{~cm}^{-1}$ for- $\mathrm{OH}$ stretch of the carboxylic acid group when complexed with HP- $\beta$-CD in the absence as well as the presence of tween 80 . The carbonyl peak shifted to a higher frequency of $1700 \mathrm{~cm}^{-1}$ and-OH stretch to a lower frequency of 3390 $\mathrm{cm}^{-1}$. This phenomenon was accompanied by peak broadening of both these peaks. The shift in the peak position was more prominent in the ternary complex as compared to binary system $\left(1736 \mathrm{~cm}^{-1}\right.$, $3383 \mathrm{~cm}^{-1}$ ), which confirms that polymer enhances the complexation efficiency of cyclodextrin with the drug.

\section{Nuclear magnetic resonance spectroscopy (NMR)}

The chemical shift variations of host or guest provide evidence for the formation of inclusion complexes in solution. The variations in the chemical shift were observed in the spectra of TEL upon complexation with $\mathrm{CD}$ and are reported in table 1. Upfield shifts were detected in the aromatic region of the spectra of TEL after complexation. The variations are more pronounced in this region owing to the interaction with $\mathrm{CD}$ protons suggesting that the biphenyl carboxylic acid moiety, as well as imidazole moiety, can be involved in the complex formation with CD.

Table 1: Variation of ${ }^{1} \mathrm{H}$ chemical shifts before and after inclusion

\begin{tabular}{ll}
\hline Protons & Shift \\
\hline H-7 & Upfield \\
H-8 & Downfield \\
H-9 & Downfield \\
H-10 & Downfield \\
H-13 and H-17 & Upfield \\
H-14 and H-16 & Upfield \\
H-19 and H-22 & Downfield \\
H-20 and H-21 & Downfield \\
\hline
\end{tabular}

Besides this, the upfield shift was also observed in the $\mathrm{H}-3^{\prime}$ and $\mathrm{H}-5^{\prime}$ protons of HP- $\beta-C D$. The magnetic anisotropic shielding induced by the 'ring-current' effect of aromatic protons results in the upfield shift of CD protons. Shifts to the higher fields of the protons located within the CD cavity suggest that a hydrophobic interaction is predominant between the drug and the $\mathrm{CD}$.

Thus 1D NMR suggests that interaction prevails between the aromatic protons and CD cavity, however, this does not confirm the formation of any complexes.
The 1D NMR results are supplemented by 2D COESY NMR which shows intermolecular dipolar cross-correlations providing important information about the evident spatial proximities between protons of TEL and HP- $\beta$-CD molecule (fig. 7). Two intermolecular cross peaks were found to be of interest between $\mathrm{H}$ $3^{\prime}$ and $\mathrm{H}-5^{\prime}$ protons of HP- $\beta-\mathrm{CD}$ with aromatic protons of TEL. These correlations indicate the spatial contacts between the wider side of the cyclodextrin cavity and the aromatic ring of TEL. The 1D and 2D NMR demonstrate the formation of inclusion complexes in a solution state. 


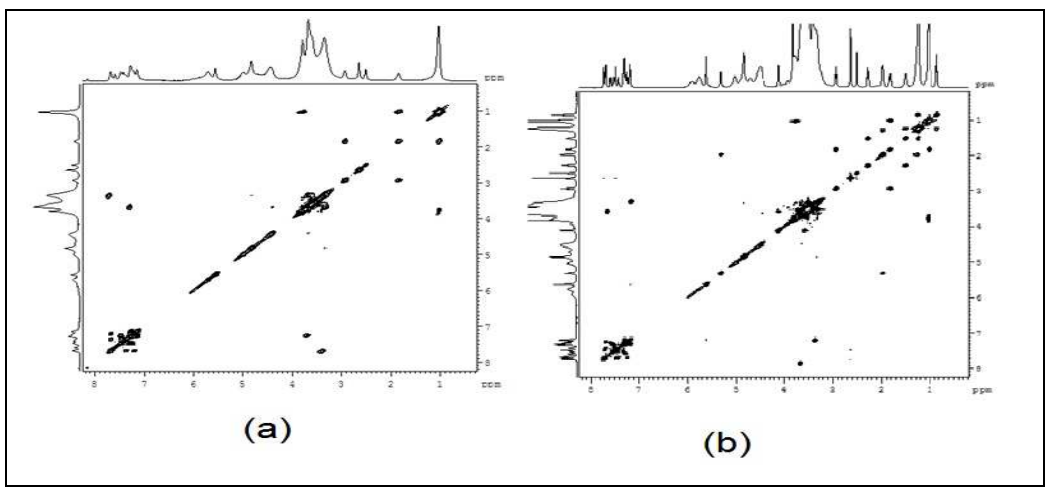

Fig. 7: COESY spectrum of (a) telmisartan with hydroxyl-propyl- $\beta$-cyclodextrin (b) telmisartan with hydroxyl-propyl- $\beta$-cyclodextrin: tween 80

The NMR studies revealed that there are two distinct regions of TEL (biphenyl carboxylic acid moiety and imidazole ring) which could be included in HP- $\beta$-CD; however, the stoichiometry of 1:1 has been established by phase solubility studies. To unravel the incongruity in the results of phase solubility and NMR and molecular modelling studies, the solution calorimetric analysis has been performed to confirm the stoichiometry and as well as to calculate the stability constant.

\section{Solution calorimetry}

By determining the enthalpy of solution, solution calorimetry was used to determine the magnitude of stability constant and enthalpy associated with binding [27]. The enthalpy of solution of TEL $(\Delta \mathrm{solH})$ determined in buffered aqueous solution was found to be- $4.53 \mathrm{~kJ} / \mathrm{mol}$ indicating an exothermic reaction. The enthalpy of solution of TEL $(\Delta$ solH $(C D))$ in the presence of cyclodextrins $(\Delta \operatorname{solH}(M)(C D))$ was found to be more exothermic which is attributed to the interaction between drug and cyclodextrin (table 2). The enthalpies of interaction per mole as well as per liter have been calculated by using Eq. 2 and 3 as discussed by Chadha et al. [32]. The stoichiometry was ascertained utilizing continuous variation method (Job's plot) by plotting ( $\Delta H$ int (M)) against $(x 2)$ for both binary and ternary systems (fig. 8). It is clearly indicated that the minima occur at $\mathrm{x}_{2}=0.5$ confirming the1:1stoichiometry as indicated by phase solubility.

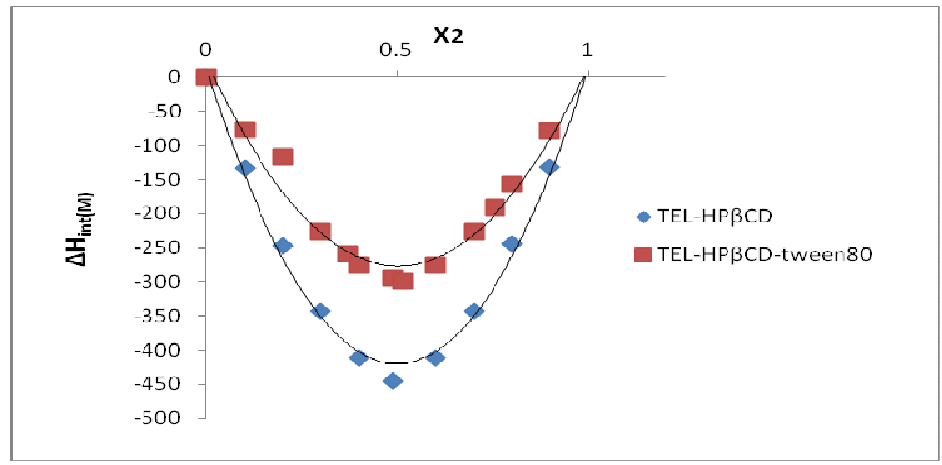

Fig. 8: Plot of molar enthalpy of interaction $\left(\Delta_{\mathrm{sol}} \mathrm{H}_{\mathrm{int}(\mathrm{M})}\right)$ of telmisartan with hydroxyl-propyl- $\beta$-cyclodextrin in the presence and absence of tween $80(0.1 \% \mathrm{v} / \mathrm{v})$

As discussed above NMR as well as molecular modelling data indicate two potential regions in TEL (imidazole ring and biphenyl carboxylic acid moiety), therefore, a two class binding model was utilized to determine the concentration of drug: CD complex which suggested that two types of 1:1 complexes co-exist in the solution. Now the binding constants are calculated assuming an equilibria between the cyclodextrin and the complex where imidazole ring enters the cyclodextrin cavity and between cyclodextrin and the complex where the biphenyl acetic acid moiety enters the host cavity. $K_{1}$ and $K_{2}$ are the equilibrium constants for both types of complexes, respectively.

Table 2: Enthalpy of solution under different initial concentration of telmisartan and hydroxyl-propyl- $\beta$-cyclodextrin and the derived quantities in binary and ternary systems

\begin{tabular}{|c|c|c|c|c|c|c|}
\hline \multirow[t]{2}{*}{$\mathbf{X}_{2}$} & \multirow[t]{2}{*}{$\mathbf{M}_{\text {VAL }}$} & \multirow[t]{2}{*}{$\mathbf{M}_{\mathrm{M}-\boldsymbol{\beta}-\mathrm{CD}}$} & \multicolumn{2}{|c|}{ For binary system } & \multicolumn{2}{|c|}{ For ternary system } \\
\hline & & & $\Delta H_{\text {int(exp) }}(\mathrm{J} / \mathrm{l})$ & $\Delta \mathrm{H}_{\text {int(M) }}\left(\mathrm{kJ} \mathrm{mol}^{-1}\right)$ & $\Delta H_{\text {int(exp) }}(\mathrm{J} / \mathrm{l})$ & $\Delta \mathrm{H}_{\mathrm{int}(\mathrm{M})}\left(\mathrm{kJ} \mathrm{mol}^{-1}\right)$ \\
\hline 0.09999 & 0.0004 & 0.0036 & -0.5314 & -132.837 & -0.30884 & -77.2023 \\
\hline 0.200184 & 0.0008 & 0.003196 & -0.9892 & -247.528 & -0.46912 & -117.388 \\
\hline 0.299898 & 0.0012 & 0.002801 & -1.37468 & -343.553 & -0.90452 & -226.053 \\
\hline 0.400276 & 0.0016 & 0.002397 & -1.64352 & -411.164 & -0.97898 & -258.592 \\
\hline 0.489922 & 0.002 & 0.002082 & -1.81892 & -445.565 & -1.09832 & -274.77 \\
\hline 0.600276 & 0.0024 & 0.001598 & -1.64352 & -411.069 & -1.20236 & -294.531 \\
\hline 0.69944 & 0.0028 & 0.001203 & -1.37496 & -343.465 & -1.2758 & -298.304 \\
\hline 0.800184 & 0.0032 & 0.000799 & -0.97478 & -243.751 & -1.09832 & -274.706 \\
\hline 0.899074 & 0.0036 & 0.000404 & -0.52952 & -132.243 & -0.90656 & -226.459 \\
\hline 0.09999 & 0.0004 & 0.0036 & -0.5314 & -132.837 & -0.75662 & -190.252 \\
\hline 0.200184 & 0.0008 & 0.003196 & -0.9892 & -247.528 & -0.62912 & -157.316 \\
\hline 0.299898 & 0.0012 & 0.002801 & -1.37468 & -343.553 & -0.3119 & -77.8948 \\
\hline
\end{tabular}


The experimentally calculated enthalpy of interaction $(\Delta H \operatorname{int}(\exp ))$ is proportional to the sum of the product of molar concentration of TEL: HP- $\beta$-CD complex in the solution at equilibrium and associated molar enthalpy of binding.

$$
\Delta \mathrm{H} \text { int (calc) }=\left[\Delta \mathrm{H}_{1} \mathrm{xK}_{1}(\mathrm{C})(\mathrm{D})\right]+\left[\Delta \mathrm{H}_{2} \times \mathrm{K}_{2}(\mathrm{C})(\mathrm{D})\right] . .
$$

Where, $\Delta H_{1}$ and $\Delta H_{2}$ are molar enthalpy of binding of complex1 and complex 2 , respectively.

$C=$ free concentration of $2-H P-\beta-C D, D=$ free concentration of TEL.

Successive iteration was used to compute the interaction parameters $(K(\mathrm{i})$ and $\Delta \mathrm{H}(\mathrm{i}))$ for the drug-CD system.
The values of $K_{1}$ and $K_{2}, \Delta H_{1}$ and $\Delta H_{2}$ were evaluated by using a selfconsistent iterative nonlinear least square regression programme after successive iteration to minimize the values of $\sum\{\Delta H$ int (exp)$\Delta H$ int L(calc) $\left.\}^{2}\right]$.

Further, the values of $C$ and $D$ were calculated by iteration until their values within selected limits were obtained by putting any reasonable initial values of $K_{1}$ and $K_{2}$. The details are given in our previous paper [32]. The values of free energy of inclusion $\left(\Delta G{ }^{\circ}{ }_{1}\right.$ and $\left.\Delta \mathrm{G}{ }^{\circ}{ }_{2}\right)$ and entropy of inclusion $\left(\Delta \mathrm{S}^{\circ}{ }_{1}\right.$ and $\left.\Delta \mathrm{S}^{\circ}{ }_{2}\right)$ for TEL have been calculated from the following equations 5-8 in the work by chadha et al., and are given in table 3.

Table 3: Thermodynamic parameters associated with the inclusion of telmisartan and hydroxyl-propyl- $\beta$-cyclodextrin in the absence and presence of tween 80 at $310 \mathrm{~K}$

\begin{tabular}{|c|c|c|c|c|c|c|c|c|}
\hline & $\begin{array}{l}K_{1} \\
\left(M^{-1}\right)\end{array}$ & $\begin{array}{l}\mathbf{K}_{2} \\
\left(\mathbf{M}^{-1}\right) \\
\end{array}$ & $\begin{array}{l}\Delta \mathrm{H}_{1}^{\circ} \\
(\mathrm{kJ} / \mathrm{mol})\end{array}$ & $\begin{array}{l}\Delta \mathrm{H}_{2}^{\circ} \\
(\mathrm{kJ} / \mathrm{mol})\end{array}$ & $\begin{array}{l}\Delta \mathrm{G}_{1}^{\circ}{ }^{\circ} \\
(\mathrm{kJ} / \mathrm{mol})\end{array}$ & $\begin{array}{l}\Delta \mathrm{G}_{2}^{\circ} \\
(\mathrm{kJ} / \mathrm{mol})\end{array}$ & $\begin{array}{l}\Delta S_{1}^{\circ} \\
(\mathrm{J} / \mathrm{mol})\end{array}$ & $\begin{array}{l}\Delta S_{2}^{\circ} \\
(J / m o l)\end{array}$ \\
\hline TEL-HP- $\beta$-CD complex & $1215 \pm 5$ & $1716 \pm 4$ & $-7.80 \pm 0.07$ & $-9.45 \pm 0.05$ & $-18.30 \pm 0.24$ & $-19.19 \pm 0.08$ & $33.89 \pm 1.26$ & $31.45 \pm 0.94$ \\
\hline $\begin{array}{l}\text { TEL-HP- } \beta \text {-CD-tween } 80 \\
\text { complex }\end{array}$ & $1699 \pm 6$ & $2088 \pm 6$ & $-12.45 \pm 0.08$ & $-13.07 \pm 0.02$ & $-19.17 \pm 0.15$ & $-19.70 \pm 0.09$ & $21.76 \pm 1.10$ & $19.35 \pm 1.02$ \\
\hline
\end{tabular}

The same method was utilized to determine the stability constant and other thermodynamic parameters in the presence of tween 80 $(0.1 \% \mathrm{v} / \mathrm{v})$. The enthalpy of solution of TEL in the presence of Tween 80 and HP- $\beta$-CD was found to be more exothermic. This is attributed to positive interaction among all the three components (TEL: HP- $\beta$-CD: tween 80 ) in the complex.

The above mentioned thermodynamic parameters, $\mathrm{K}, \Delta \mathrm{H}, \Delta \mathrm{G}$ and $\Delta \mathrm{S}$, of the binding process are essential for determining the driving forces of interaction of TEL with HP- $\beta$-CD. The values of the binding constants thus determined for both binary and ternary systems are given in table 4 . The higher value of $\mathrm{K}\left(\mathrm{K}_{1}: 1888 \mathrm{M}^{-1}\right.$ and $\left.\mathrm{K}_{2}: 1699 \mathrm{M}^{-1}\right)$ for ternary system reflects the higher efficiency of TEL to be included into the cyclodextrin cavity. This synergistic effect shows that tween 80 facilitates the inclusion of TEL into HP- $\beta$-CD cavity. The negative values for free energy for both binary and ternary complexes means that the binding process is a spontaneous process and thermodynamically favored. The entropic terms, $\Delta S>0$, for the complexation are also found to be favourable for complexation. The standard enthalpy of formation of both binary and ternary complexes was found to be negative indicating that the formation of the inclusion complex is an exothermic process.

This exothermic energy may be the outcome of the release of water molecules into the aqueous phase from the completely hydrated cavity of the $\mathrm{CD}$ and the hydrophobic interactions between the host and the guest.

The negative standard Gibb's free energy indicates the complexation is a spontaneous process and the value is more negative in ternary complex indicating that the process is thermodynamically favoured.

The entropy of both the systems is positive and thus it makes a larger contribution to the negative Gibbs free energy instead of enthalpy. This suggests that the binding process is entropically driven.

\section{Dissolution studies}

The percent release of TEL from the physical mixture, TEL complexes with HP- $\beta$-CD in the presence and absence of tween 80 was compared by using two relative parameters. At time $t=2 \mathrm{~min}$ the two relative parameters of the dissolution process, i.e. the percentage of TEL dissolved (DP) and the relative dissolution rate (RDR) for pure drug, TEL: HP- $\beta$-CD (physical mixture), binary and ternary system, respectively, were calculated and followed the order as TEL: HP- $\beta$-CD: tween $80(0.1 \%$ v/v)>TEL: HP- $\beta$-CD>physical mixture>TEL (fig. 9). There is 6 folds increase in TEL dissolution rate from the ternary complex as compared to that from the pure drug.

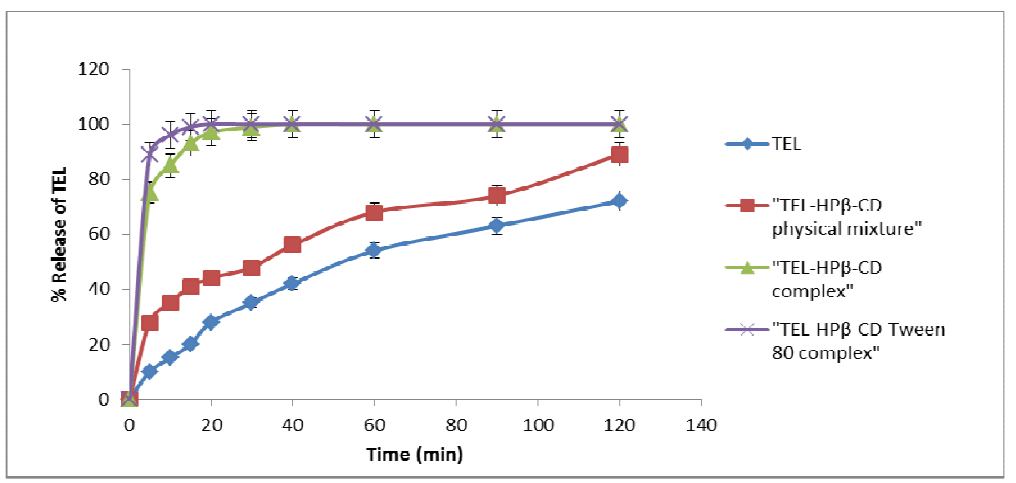

Fig. 9: Dissolution profiles of telmisartan, physical mixture, binary system and ternary system $(n=3)$, significant increase in $\%$ release of telmisartan-HP- $\beta C D$ physical mixture, telmisartan-HP- $\beta C D$ complex, telmisartan-HP- $\beta C D-T$ ween 80 complex compared to telmisartan $(P \leq 0.005)$

\section{Pharmacological studies}

On contrary to control rats (group I and II) a gradual noticeable increase of approximately $50-90 \mathrm{~mm} \mathrm{Hg}$ in mean systolic blood pressure over a period of six weeks was observed in DOCA treated rats (group III, IV, V and VI).
After the oral administration of HP- $\beta-C D, H P-\beta-C D$ : tween 80 and CMC suspension to groups I and II, changes in the systolic blood pressure were recorded and expressed as the percentage decrease in the systolic blood pressure. No noteworthy change in the blood pressure levels of rats during the experimental period was observed (fig. 10). 


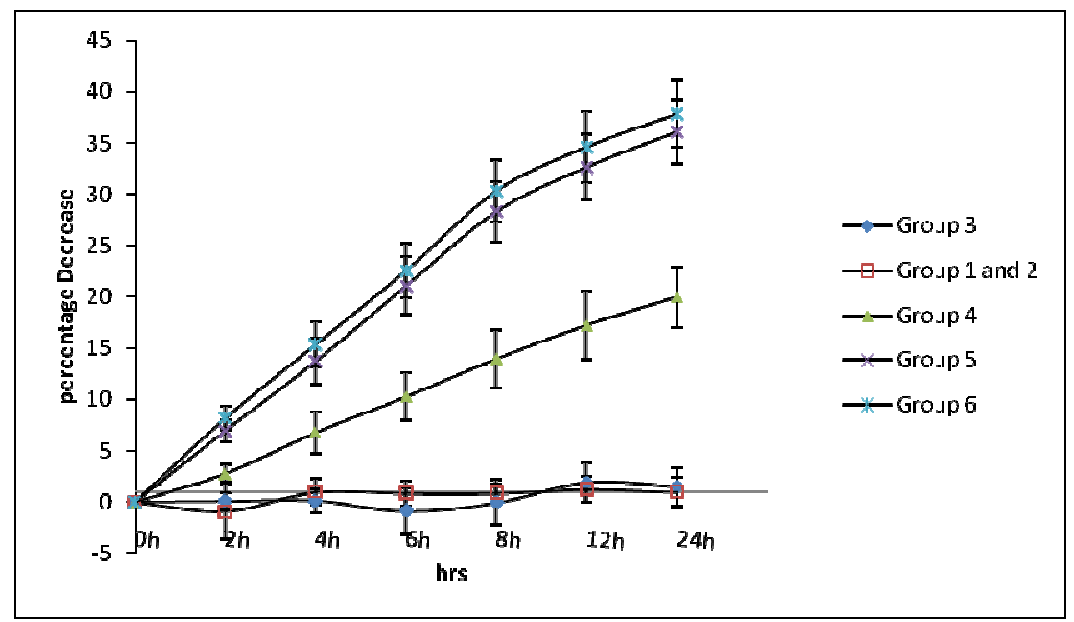

Fig. 10: Effect of telmisartan on blood pressure in DOCA induced hypertension (n=6), (Significant decrease in blood pressure as compared to control, DOCA control, binary and ternary system groups $(P \leq 0.001)$

During the experimental period after six weeks the variation in systolic blood pressure caused by oral administration of TEL, TEL: HP- $\beta$-CD, TEL: HP- $\beta$-CD: tween 80 to groups III, IV and V has been depicted in fig. 10. All they had significantly lowered the elevated systolic blood pressure in hypertensive rats as compared to vehicle treated DOCA control rats. TEL has reduced the systolic blood pressure but was not able to normalize whereas the TEL: HP- $\beta-C D$, TEL: HP- $\beta$-CD: tween 80 had normalized the increased blood pressure values in hypertensive rats. After seven day's treatment with TEL: HP- $\beta$-CD: tween 80 , the percentage decrease of $37.28 \%$ in the elevated systolic blood pressure values was observed while TEL: HP- $\beta$-CD and TEL showed 31.25 and $19.39 \%$, respectively. From these data, it was also indicated that inclusion complexation of TEL with HP- $\beta-C D$ in the presence as well as the absence of tween 80 has increased the drug performance as shown by the higher percentage decrease in blood pressure values than TEL alone due to increase in the solubility of TEL in presence of cyclodextrin.

The experimental data depicted in fig. 10 was statistically analysed by ANOVA and a significant decrease in systolic blood pressure as compared to the control, DOCA control and the pure drug was observed $(\mathrm{P} \leq 0.001)$.

In the above work, the phase solubility studies suggest a 1:1 stoichiometry between TEL and HP- $\beta$-CD which is not affected by the presence of tween 80. The disappearance of characteristic drug peaks in both DSC and PXRD studies suggest the complete inclusion of drug into cyclodextrin. The molecular modelling and NMR studies support the hypothesis of 1:1 stoichiometry between drug and cyclodextrin which is confirmed by solution calorimetric technique. Kane et al. have also used cyclodextrins as the means to improve solubility of telmisartan, however, the authors have not confirmed the stoichiometry of complex formed as is done in the present work [34]. The high binding and thermodynamic parameters illustrate that presence of tween 80 facilitates the inclusion. A synergistic effect has been observed in the presence of tween 80 . The enhancement in bioavailability of TEL was the result of increased solubility and dissolution profile of TEL complexed with HP- $\beta$-CD in the absence and presence of tween 80. Muankaew et al. have prepared a cyclodextrin based formulation of telmisartan using $\gamma$ cyclodextrin as binary component and hydroxyl propyl methyl cellulose as the ternary component. The authors have also found that there is an increase in solubility and dissolution of telmisartan in the complexed form [35].

\section{CONCLUSION}

The complexation efficiency of HP- $\beta-C D$ with TEL was found to increase on the addition of $0.1 \% \mathrm{v} / \mathrm{v}$ tween 80 . Solution calorimetry established the NMR and molecular modelling results which showed the simultaneous existence of two types of complexes. In the presence of third component [tween $80(0.1 \% \mathrm{v} / \mathrm{v})$ ] higher values of stability constants were obtained and also the negative enthalpy and positive entropy of binding, determined by solution calorimetry, indicated the formation of the stable system. The dissolution rate for ternary was found to be higher than the binary system and pure drug. The maximum decrease in systolic blood pressure in DOCA induced rats were observed for this system, indicating the improvement of the pharmaceutical potential of TEL in the form of TEL: HP- $\beta$-CD: tween $80(0.1 \% \mathrm{v} / \mathrm{v})$ complex.

\section{AUTHOR CONTRIBUTION}

All the authors have contributed in the completion of this research work.

\section{ACKNOWLEDGEMENT}

The authors would like to appreciate the financial assistance provided by University Grant Commission, New Delhi, India and the computational facilities supported by the Department of Biotechnology (DBT; BT/TF8/BRB/2009) and CSIR (01/2399/10/EMRII 576and 02(0047)/12/ EMR-II), New Delhi, India.

\section{CONFLICTS OF INTERESTS}

The authors report no conflicts of interest. The authors alone are responsible for the content and writing of the article.

\section{REFERENCES}

1. Davis ME, Brewster ME. Cyclodextrin-based pharmaceutics: the past, present and future. Nat Rev Drug Discovery 2005;12:1023-35

2. Loftsson T, Pekka J, Mar M, Tomi J. Cyclodextrins in drug delivery. Macroc Chem 2002;44:213-8.

3. Martin Del VEM. Cyclodextrins and their uses: a review. Process Biochem 2004;39:1033-46.

4. Yadav N, Chhabra G, Pathak K. Enhancement of solubility and dissolution rate of a poorly water soluble drug using single and double hydrophilization approach. Int J Pharm Pharm Sci 2012;4:975-1491.

5. Thompson DO. Cyclodextrins enabling excipients: their present and future use in pharmaceuticals. Crit Rev Ther Drug 1997;14:1-104.

6. Stella VJ, Rajewski R. Cyclodextrins: Their future in drug formulation and delivery. Pharm Res 1997;14:556-67.

7. Heise HM, Kuckuk R, Bereck A, Riegel D. Infrared spectroscopy and Raman spectroscopy of cyclodextrin derivatives and their ferrocene inclusion complexes. Vib Spectrosc 2010;53:19-23.

8. Smith JS, Macrae RJ, Snowden MJ. Effect of SBE7- $\beta$-cyclodextrin complexation on carbamazepine release from sustained release beads. Eur J Pharm Sci 2005;60:73-80.

9. Mendhe AA, Kharwade RS, Mahajan UN. Dissolution enhancement of poorly water-soluble drug by cyclodextrins inclusion complexation. Int J Appl Pharm 2016;8:60-5. 
10. Lakshmana PVR, Rambabu C. Use of ion association complex formation for the spectrophotometric determination of itopride HCL in bulk and its pharmaceutical preparations. Int J Curr Pharm Res 2017;9:81-4.

11. Sarangi MK, Padhi S. Colon targeted drug delivery system-an approach for treating colonic ailments. J Crit Rev 2015;2:12-8.

12. Harika K, Sunitha K, Pavan Kumar P, Madhusudan Rao Y. Influence of hydroxypropyl- $\beta$-cyclodextrin on repaglinide release from sustained release bio adhesive buccal tablets. Asian J Pharm Clin Res 2013;6:184-90.

13. Al-Nuss Raghad, El-Zein Hind. Enhancement of candesartan cilexetil dissolution rate by using different methods. Asian J Pharm Clin Res 2015;8:320-6.

14. Loftsson T, Fridriksdóttir H. The effect of water-soluble polymers on the aqueous solubility and complexing abilities of $\beta$-cyclodextrin. Int J Pharm 1998;163:115-21.

15. Lahiani M, Barbot C, Bounoure F, Joudieh S, Skiba M. Solubility and dissolution rate of progesterone-cyclodextrin-polymer systems. Drug Dev Ind Pharm 2006;32:1043-58.

16. Ribeiro LSS, Ferreira DC, Veiga FJB. Physicochemical investigation of the effects of water-soluble polymers on vipocetine complexation with $\beta$-cyclodextrin and its sulfonyl ether derivative in solution and solid state. Eur J Pharm Sci 2003;20:253-66.

17. Lofttson T, Hreinsdóttir D, Másson M. Evaluation of cyclodextrin solubilization of drugs. Int J Pharm 2005b;302:18-28.

18. Messner M, Kurkov SV, Flavia`-Piera R, Brewster ME, Loftsson T. Self-assembly of cyclodextrins: the effect of the guest molecule. Int J Pharm 2011;408:235-47.

19. Becket G, Schep LJ, Tan MY. Improvement of the in vitro dissolution of praziquantal by complexation with alpha-, beta and gamma cyclodextrins. Int J Pharm 1999;179:65-71.

20. Challa R, Ahuja A, Ali J, Khar R. Cyclodextrin in drug delivery: an updated review. AAPS Pharm SciTech 2005;6:329-57.

21. Castagne D, Dive G, Evrard B, Fre'de'rich M, Piel G. Spectroscopic studies and molecular modelling for understanding the interactions between cholesterol and cyclodextrins. J Pharm Pharm Sci 2010;13:362-77.

22. Kang J, Kumar V, Yang D, Chowdhury PR, Hohl RJ. Cyclodextrin complexation: influence on the solubility, stability, and cytotoxicity of camptothecin, an antineoplastic agent. Eur J Pharm Sci 2002;15:163-70.

23. Londhe V, Nagarsenker M. Comparision between Hydroxypropyl- $\beta$-cyclodextrin and polyvinyl pyrrolidine as carriers for carbamazepine solid dispersions. Indian J Pharm Sci 1999;61:237-40.

24. Nalawade P, Aware B, Dand N, Kadam V, Hirlekar R. Solid state characterization of the inclusion complex of valsartan with methyl- $\beta$-cyclodextrin. J Inclusion Phenom Macrocyclic Chem 2009;65:377-8.

25. Carrier RL, Miller LA, Ahmed I. The utility of cyclodextrins for enhancing oral bioavailability. J Controlled Release 2007;123: 78-99.

26. Loftsson T, Sigurdardóttir AM. The effect of hydroxypropyl methylcellulose on hydroxypropyl- $\beta$-cyclodextrin complexation of hydrocortisone and its permeability through hair mouse skin. Eur J Pharm Sci 1994;2:297-301.

27. Loftsson T, Vogensen S, Brewster ME, Konraosdottir F. Effects of cyclodextrins on drug delivery through biological membranes. J Pharm Sci 2007;10:2532-46.

28. Uekama K. Design and evaluation of cyclodextrin based drug formulation. Chem Pharm Bull 2004;8:900-15.

29. Higuchi T, Connors KA. Phase solubility techniques. Adv Anal Chem Instrum 1965;4:117-22.

30. Chadha R, Arora P, Gupta S, Jain DVS. Complexation of nevirapine with cyclodextrins in the presence and absence of tween 80:Characterization, thermodynamic parameters, and permeability flux. J Therm Anal Calorin 2011;105:1049-59.

31. Chadha R, Jain DVS, Aggarawal A, Singh S, Thakur D. Binding constants of inclusion complexes of nitroimidazoles with $\beta$ cyclodextrins in the absence and presence of PVP. Thermochim Acta 2007;459:111-5.

32. Chadha R, Gupta S, Shukla G, Jain DVS, Pissurlenkar RRS, Coutinho EC. Interaction of artesunate with $\beta$ cyclodextrins: Characterisation, thermodynamic parameters, molecular modelling, the effect of PEG on complexation and antimalarial activity. Results Pharma Sci 2011;1:38-48.

33. Grillo R, De Melo NFS, Morales Moraes C, De Limba R, Menezes CMS, Ferreira EI, et al. Study of the interaction between hydroxymethyl nitrofurazone and 2-hydroxy- $\beta$-cyclodextrin. J Pharm Biomed Anal 2008;47:295-302.

34. Kane RN, Kuchekar BS. Preparation, physicochemical characterization, dissolution and formulation studies of telmisartan cyclodextrin inclusion complexes. Asian J Pharm 2010;4:52-9.

35. Muankaew C, Jansook P, Sigurđsson HH, Loftsson T. Cyclodextrin-based telmisartan ophthalmic suspension: formulation development for water-insoluble drugs. Int J Pharm 2016;30:21-31.

\section{How to cite this article}

- Poonam Arora, Jaspreet Singh, Renu Chadha. Physicochemical characterization and evaluation of telmisartan: hydroxypropyl- $\beta$ cyclodextrin: tween 80 inclusion complex. Int J Pharm Pharm Sci 2017;9(9):51-58. 